\title{
Pseudorecombination between Two Distinct Strains of Cucumber mosaic virus Results in Enhancement of Symptom Severity
}

\author{
Mi Sa Vo Phan', Jang-Kyun Seo ${ }^{2}$, Hong-Soo Choi ${ }^{2}$, Su-Heon Lee ${ }^{3}$ and Kook-Hyung Kim ${ }^{1,4 *}$ \\ ${ }^{1}$ Department of Agricultural Biotechnology and Plant Genomics and Breeding Institute, Seoul National University, Seoul \\ 151-921, Korea \\ ${ }^{2}$ Crop Protection Division, National Academy of Agricultural Science, Rural Development Administration, Suwon 441-707, \\ Korea \\ ${ }^{3}$ Department of Applied Biology, Kyungpook National University, Daegu 702-701, Korea \\ ${ }^{4}$ Research Institute of Agriculture and Life Sciences, Seoul National University, Seoul 151-921, Korea
}

(Received on April 10, 2014; Accepted on May 8, 2014)

Recently, a Cucumber mosaic virus (CMV) strain, named as CMV-209, was isolated from Glycine soja. In this study, symptom expression of CMV-209 was analyzed in detail in Nicotiana benthamiana by comparing with that of CMV-Fny, which is a representative strain of CMV. Using infectious cDNA clones of CMV strains 209 and Fny, symptom expression of various pseudorecombinants between these two strains were examined in the early and late infection stages. In the early infection stage, the pseudorecombinants containing Fny-RNA2 induced stunting and leaf distortion on the newly emerged leaves whereas the pseudorecombinants containing 209-RNA2 caused no obvious symptoms. In the late infection stage, the pseudorecombinants containing 209-RNA1 and Fny-RNA2 induced severe leaf distortion and stunting, while CMV-209 induced mild symptom and CMV-Fny caused typical mosaic, general stunting, and leaf distortion symptoms, indicating that RNA 2 encodes a symptom determinant(s) of CMV, which is capable of enhancing symptoms. Furthermore, our results support the possibility that natural recombination between compatible viruses can result in emergence of novel viruses causing severe damages in crop fields.

Keywords : Cucumber mosaic virus, symptom expression, pseudorecombination

*Corresponding author.

Phone) +82-2-880-4677, FAX) +82-2-873-2317

E-mail)kookkim@snu.ac.kr
Cucumber mosaic virus (CMV) is an economically important plant pathogen all over the world and has a broad host range of more than 1,200 plant species (Palukaitis and García-Arenal, 2003). The CMV genome comprises of three single-stranded plus-sense RNAs (Palukaitis et al., 1992). RNA1 encodes one large 1a protein which contains a putative methyltransferase domain (Mi et al., 1989; Mi and Stollar, 1991; Rozanov et al., 1992) and helicase motif (Gorbalenya et al., 1989; Habili and Symons, 1989; Hodgman, 1988). RNA2 encodes the $2 \mathrm{a}$ protein containing the GDD motif typical for RNA-dependent RNA polymerase (RdRp) (Hayes and Buck, 1990) and one small 2b protein which has a function of a silencing suppressor (Brigneti et al., 1998; Lucy et al., 2000). RNA3 encodes the viral movement protein (3a protein) and the capsid protein (CP) (Davies and Symons, 1988; Ding et al., 1995a; Kaplan et al., 1995). The $2 \mathrm{~b}$ protein and CP are translated from the subgenomic RNA4A (Ding et al., 1994) and RNA4 (Schwinghamer and Symons, 1975), respectively.

The specific strains of CMV have been characterized to induce various symptoms on different hosts (Palukaitis et al., 1992). In tobacco, most strains of CMV induced a light-green or dark-green mosaic (Takanami, 1981). However, some strains induced distinct symptoms such as leaf distortion and stunting (Szilassy et al., 1999), bright yellow chlorosis (Fulton, 1950; Takanami, 1981), or necrotic lesions (Divéki et al., 2004; Troutman and Fulton, 1958; Zhang et al., 1994). To date, symptom determinants of CMV have been analyzed extensively and almost the location of these symptom determinants were either on RNA3 (Shintaku, 1991; Shintaku et al., 1992; Suzuki et al., 1995; Szilassy et al., 1999; Zhang et al., 1994) or RNA2 (Ding et al., 1995b; Ding et al., 1996; Du et al., 2007; Du et al., 2008; Shi et al., 2002; Soards et al., 2002; Ziebell et 
Table 1. Primers used for CMV-detection and construction of the CMV cDNA clones

\begin{tabular}{ll}
\hline Name & Sequence $\left(5^{\prime} \rightarrow 3^{\prime}\right)^{\mathrm{a}}$ \\
\hline CMV-3-end-SmaI & GCCCGGGTGGTCTCCTTTTRGAGRCC \\
CMV-R1R2-5-end-T7 & taatacgactcactataGTTTATTTACAAGAGCGTACGG \\
CMV-R3-5-end- $T 7$ & taatacgactcactataGTAATCTTACCACTGTGTGTGTG \\
CMV-detR1-F & TTTTTGAACCGTCCACTGACATGA \\
CMV-detR1-R & AGTGAAGCCTTATCGGCTTGGG \\
\hline
\end{tabular}

${ }^{\mathrm{a}}$ The SmaI sequence is shown in bold face and the sequence of $\mathrm{T} 7$ promoter is written in italic.

al., 2007). In a few cases, the determinants of expression symptom has been mapped to RNA1 (Divéki et al., 2004) or both RNA1 and RNA2 (Zhang et al., 1994).

CMV-209 is a CMV strain, which was recently isolated from Glycine soja (Vo Phan et al., 2014). In this study, symptom expression of CMV-209 was analyzed in Nicotiana benthamiana based on pseudorecombination of genomic RNAs of CMV-209 with those of CMV-Fny, which is a typical strain of CMV. Our results demonstrated that, in the early infection stage, the pseudorecombinants harboring Fny-RNA2 caused stunting and leaf distortion on the newly emerged leaves although the pseudorecombinants holding 209-RNA2 exhibited no obvious symptoms. In the late infection stage, the pseudorecombinants consisting of 209-RNA1 and Fny-RNA2 induced severe leaf distortion and stunting, while CMV-209 induced mild symptom and CMV-Fny caused typical mosaic, general stunting, and leaf distortion symptoms, indicating that the symptom determinant(s) of CMV is included in RNA2 and is capable of enhancing symptom severity. Additionally, our results support the possibility that novel viruses causing severe damages in crop fields can be emerged by natural recombination between compatible viruses.

The full-length cDNAs of genomic RNA of CMV-209 and -Fny were amplified using the cDNA clones of CMV 209 (i.e. pCMV-209R1, pCMV-209R2 and pCMV-209R3) (Vo Phan et al., 2014) and Fny [i.e. pCR1 (+), pCR2 (+) and pCR3 $(+)$ ] (Seo et al., 2009) as templates. The primers, CMV-R1R2-5-end-T7, CMV-R3-5-end-T7, and CMV3-end-SmaI (Table 1), were used for PCR, thereby the resulting products contained a T7 promoter and SmaI site at the $5^{\prime}$ and $3^{\prime}$ ends, respectively. The resulting PCR products were cloned directly into pGEM $^{\circledR}-\mathrm{T}$ Easy vector (Promega, USA). The final cDNA clones of CMV-209 and -Fny constructed for in vitro transcription were named pT7CMV-209R1, pT7-CMV-209R2, pT7-CMV-209R3, pT7CMV-FnyR1, pT7-CMV-FnyR2, and pT7-CMV-FnyR3, respectively (Fig. 1).

The CMV-209 and -Fny clones were linearized by SmaI. The cDNA-derived infectious RNAs of CMV-209 and
-Fny were generated by in vitro transcription with T7 RNA polymerase (Takara, Japan) in the presence of 7-methylguanosine "cap" structure (Promega, USA) according to the manufacturer's protocol. The in vitro transcripts from the full-length cDNA clones of CMV-209 and -Fny RNAs 1,2 , and 3 were named $\mathrm{W} 1, \mathrm{~W} 2, \mathrm{~W} 3, \mathrm{~F} 1, \mathrm{~F} 2$, and F3, respectively (Fig. 1) and mixed to mimic the original CMV RNAs (i.e. CMV-209, W1W2W3; CMV-Fny, F1F2F3). Six different pseudorecombinants, W1W2F3, W1F2W3, F1W2W3, F1F2W3, F1W2F3, and W1F2F3, were also generated by mixing the in vitro transcripts. All of the parental and pseudorecombinant RNAs were diluted in the inoculation buffer ( $0.05 \mathrm{M}$ potassium phosphate, $\mathrm{pH} 7.5)$ and total $1.5 \mu \mathrm{g}$ of RNA transcripts (500 ng of each RNA transcript 1, 2 and 3) were inoculated per plant. All the $N$. benthamiana plants inoculated with transcripts were lightly dusted with carborundum before inoculation. Mocktreated plants were inoculated with the inoculation buffer. The inoculated plants were maintained in an insect-free green house at $25^{\circ} \mathrm{C}$ under a $14 \mathrm{~h}$ photoperiod. The disease

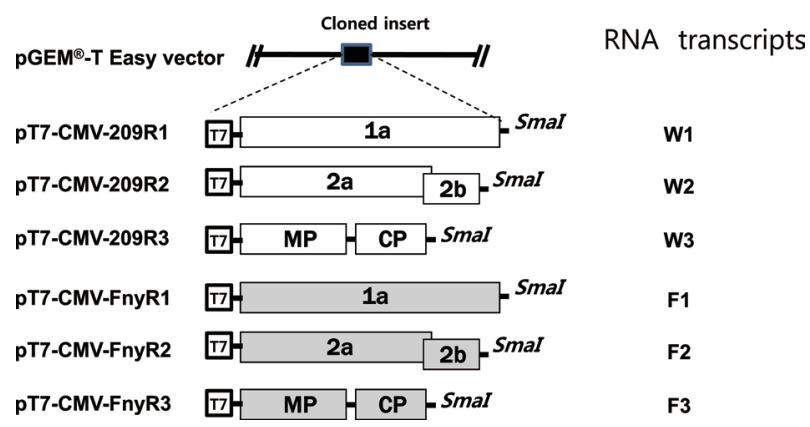

Fig. 1. Schematic diagram of construction of infectious cDNA clones of CMV-209 and CMV-Fny strains and names of their RNA transcripts. The constructs pT7-CMV-209R1, pT7-CMV209R2, pT7-CMV-209R3 and pT7-CMV-FnyR1, pT7-CMVFnyR2, pT7-CMV-FnyR3 express RNA1, RNA2, and RNA3 of CMV strain 209 and Fny, respectively. W1, W2, W3 and F1, F2, F3 are RNA transcripts synthesized from templates pT7-CMV209R1, pT7-CMV-209R2, pT7-CMV-209R3 and pT7-CMVFnyR1, pT7-CMV-FnyR2, pT7-CMV-FnyR3, respectively by in vitro transcription. 

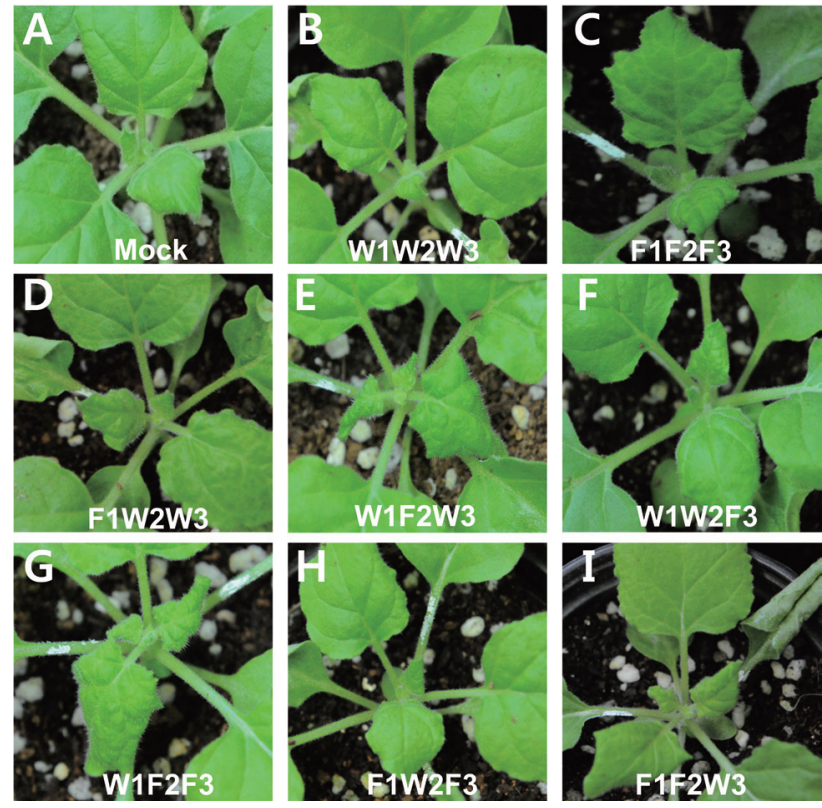

Fig. 2. Symptoms induced in Nicotiana benthamiana by CMV209, CMV-Fny, and their pseudorecombinants constructed in vitro from the three genomic segments of the two virus strains. W1W2W3 (CMV-209), W1W2F3 and F1W2F3 caused mind symptom (B, F, H, respectively). F1F2F3 (CMV-Fny) and F1F2W3 caused general stunting, vein clearing and leaf distortion (C, I, respectively). F1W2W3 induced vein clearing symptom (D). W1F2W3 and W1F2F3 induced severe stunting and leaf distortion (E, G, respectively). Mock represents healthy plant. Photographs were taken at 7 days after inoculation.

symptoms were observed at two stages, the early infection stage [7 days post inoculation (dpi)] and the late infection stage (12 dpi). CMV infection of the inoculated plants was confirmed by RT-PCR at 7 dpi using primers CMV-detR1F and CMV-detR1-R (Table 1).

The $N$. benthamiana plants infected with CMV-Fny (F1F2F3) showed general stunting, vein clearing, and leaf distortion on the infected systemic leaves in the early infection stage (Fig. 2C). In contrast, the plant inoculated with CMV-209 (W1W2W3) developed no obvious symptoms and indistinguishable from healthy plants although they were systemically infected with CMV-209 as confirmed by a specific RT-PCR (Fig. 2B and 3). Thus, we sought to examine what differences between two strains of CMV affect symptom expression. To this end, we performed inoculation assays using pseudorecombinants of CMV-Fny and-209. Six pseudorecombinants (F1W2W3, W1F2W3, W1W2F3, W1F2F3, F1W2F3, and F1F2W3) were inoculated in $N$. benthamiana. At 7 dpi, the symptoms induced in the inoculated $N$. benthamiana plants were observed in detail (Fig. 2 and Table 2). Viral infection of the inoculated

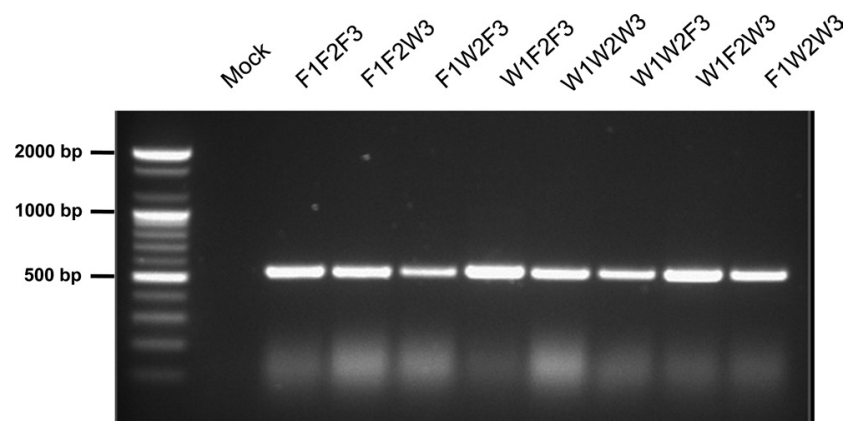

Fig. 3. Detection of CMV in $N$. benthamiana plants infected with CMV-209, CMV-Fny, and their pseudorecombinants. Total nucleic acids were extracted from upper un-inoculated leaves of infected plants at 7 days after inoculation, and subjected to RTPCR analysis using primer CMV-detR1-F and CMV-detR1-R.

plants was confirmed by RT-PCR using total RNA extracted from upper un-inoculated leaves (Fig. 3). Interestingly, pseudorecombination resulted in induction of different symptoms according to combinations of viral genomic RNAs. The pseudorecombinant of F1W2W3 induced vein clearing but no leaf distortion on the upper leaves of infected plants (Fig. 2D). The pseudorecombinants containing F2 (i.e. F1F2W3, W1F2F3, and W1F2W3) produced leaf distortion and stunting symptoms (Fig. 2E, G and I). However, two pseudorecombinants of W1W2F3 and F1W2F3 induced no obvious symptoms (Fig. 2F and H). In summary, the pseudorecombinants containing F2 induced stunting and leaf distortion on the newly emerged leaves, while those containing W2 (except for F1W2W3, which induced vein clearing) caused no obvious symptoms in the early infection stage. Therefore our results suggested that CMV RNA2 contains a symptom determinant(s) causing stunting and leaf distortion in the early infection stage.

We further observed the infected plants to investigate whether the symptoms induced by the pseudorecombinants could be intensified in the late infection stage. To this end, the $N$. benthamiana plants infected with each pseudorecombinant were observed at $12 \mathrm{dpi}$. Similar to the observation in the early infection stage, the plant inoculated with CMV-209 (W1W2W3) exhibited no obvious symptoms and indistinguishable from healthy plants (Fig. 4B), whereas CMV-Fny (F1F2F3) induced vein clearing, general leaf distortion, and severe mosaic symptoms on the infected systemic leaves at 12 dpi (Fig. 4C). The pseudorecombinants, W1W2F3 and F1W2W3, also did not cause any obvious symptoms as similar to CMV-209 (Fig. $4 \mathrm{~F}$ and $\mathrm{H})$. However, the pseudorecombinants, W1F2W3 and W1F2F3 containing CMV-Fny RNA2 induced severe leaf distortion and stunting symptoms (Fig. 4E and G). Ex- 
Table 2. Characteristics of symptoms induced by CMV-209, CMV-Fny and their pseudorecombinants on Nicotiana benthamiana plants

\begin{tabular}{|c|c|c|}
\hline \multirow[t]{2}{*}{ Inoculum $^{\mathrm{a}}$} & \multicolumn{2}{|c|}{$\begin{array}{l}\text { Disease symptom } \mathrm{b} / \text { severity of systemic }^{\text {seric }} \\
\text { symptom }^{\mathrm{c}}\end{array}$} \\
\hline & $7 \mathrm{dpi}$ & 12 dpi \\
\hline W1W2W3 & $\mathrm{ns} /-$ & $\mathrm{ns} /-$ \\
\hline W1W2F3 & $\mathrm{ns} /-$ & $\mathrm{ns} /-$ \\
\hline W1F2W3 & ld, gst/++ & sld, sst/++++ \\
\hline F1W2W3 & $\mathrm{vc} /+$ & $\mathrm{vc}, \mathrm{mld} /+$ \\
\hline F1F2F3 & $\mathrm{vc}, \mathrm{ld}, \mathrm{gst} /++$ & vc. mld, smo /+++ \\
\hline F1F2W3 & vc, ld, gst/++ & $\mathrm{vc}, \mathrm{mmo} /++$ \\
\hline F1W2F3 & $\mathrm{ns} /-$ & $\mathrm{ns} /-$ \\
\hline W1F2F3 & ld, gst/++ & sld, sst/++++ \\
\hline
\end{tabular}

${ }^{\mathrm{a}}$ Inoculums of CMV-209, CMV-Fny and their pseudorecombinants were generated by mixing of in vitro RNA transcripts.

'Symbols: ns, no obvious symptom; ld, leaf distortion; sld, severe leaf distortion; mld, mild leaf distortion; gst: general stunting; sst, severe stunting; vc, vein clearing; smo, severe mosaic; mmo; mild mosaic.

c-, no obvious symptom, + , mild symptom; ++, intermediate symptom; +++, severe symptom; ++++, very severe symptom.

ceptionally, the plant infected with F1F2W3, showed vein clearing and mild mosaic symptoms (Fig. 4I), while those infected with F1W2W3 developed vein clearing and mild leaf distortion in the late infection stage (Fig. 4D). Our results implied that the compatibility among CMV genomic RNA segments plays a role in symptom expression and, as observed in the cases of W1F2W3 and W1F2F3, pseudorecombination between distinct strains of a virus species can result in emergence of virus progenies carrying higher infectivity or causing severe damages.

In general, disease symptom development of $N$. benthamiana plant induced by CMV-Fny could be divided into two visually distinct stages. Under our plant growth condition, the newly emerged leaves after inoculation of CMV-Fny initially showed vein clearing and general stunting and leaf distortion at $7 \mathrm{dpi}$ (Early infection stage, Fig. 2C) and then the next leaves emerged at 12 dpi showed green mosaic (Late infection stage, Fig. 4C). CMV-Fny RNA1 might play a role in causing vein clearing in the early infection stage, because it was evident that the pseudorecombinants, F1W2W3 and F1F2W3 induced vein clearing while the other recombinants containing W1 did not (Fig. 2). Zhang et al. (1994) also suggested that both RNAs 1 and 2 of CMV-Fny were involved in determining the severity of systemic symptom on tobacco working with CMV-Fny and CMV-LS (Zhang et al., 1994). However, Rao and Franki (1982) handled with eighteen pseudorecombinants
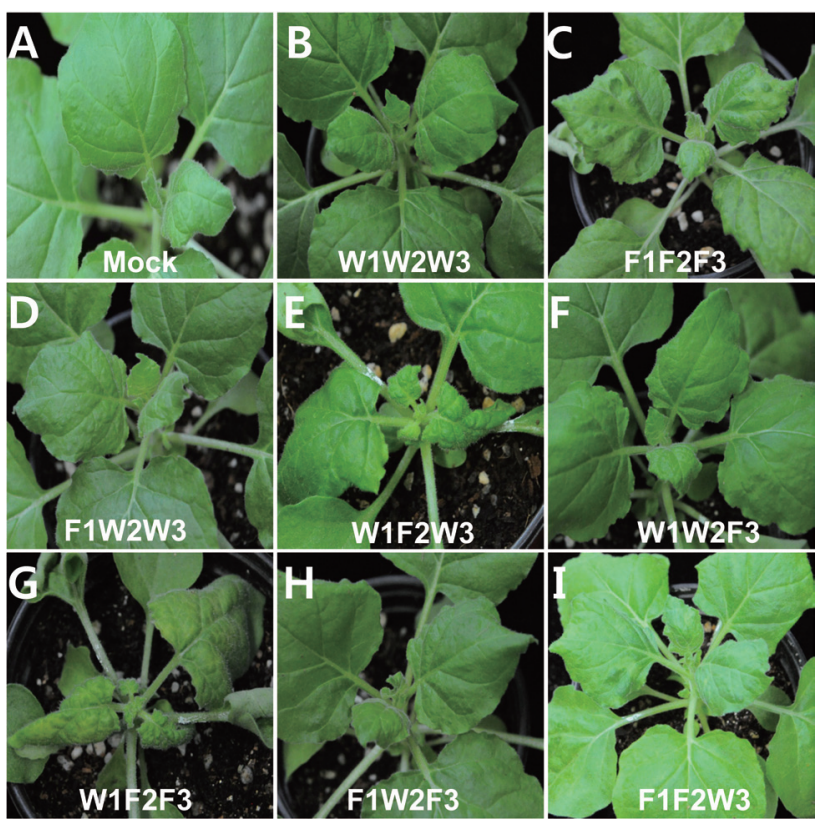

Fig. 4. Symptoms induced in N. benthamiana by CMV-209, CMV-Fny, and their pseudorecombinants constructed in vitro from the three genomic segments of the two virus strains. W1W2W3 (CMV-209), F1W2F3, W1W2F3 and F1W2F3 caused mind symptom (B, D, F, H, respectively). F1F2F3 (CMVFny) and F1F2W3 caused general stunting, mosaic, and leaf distortion (C, I, respectively). W1F2W3 and W1F2F3 induced severe stunting and leaf distortion (E, G, respectively). Mock represents healthy plant. Photographs were taken at 12 days after inoculation.

constructed in vitro from three strains of CMV (CMV-U, CMV-M, and CMV-K) and showed that RNA1 had little effect on symptom induction (Rao and Francki, 1982). The fact that involvement of CMV RNA1 in symptom determination was reported rather rare, except for mapping of the genetic determinant for necrosis of CMV-Ns to the protein 1a (Divéki et al., 2004).

Our results clearly show that CMV-Fny RNA2 played a crucial role in symptom induction in both the early and late infection stage. F1W2F3 caused no obvious symptom (Fig. 2H, 4H) while W1F2W3 induced stunting and leaf distortion (Fig. 2E, 4E) all the time during the viral infection. According to the previous reports that the mutant CMV-Fny- $\Delta 2 b$, in which the $2 b$ ORF was deleted, could move systemically in tobacco and $N$. benthamiana but could not induce symptom (Soards et al., 2002; Ziebell et al., 2007) and transgenic plants expressing the CMVFny $2 \mathrm{~b}$ protein exhibited strong symptom-like phenotypes such as leaf distortion, general stunting (Lewsey et al., 2007), CMV-Fny $2 \mathrm{~b}$ protein seemed to have ability to control symptom expression (Ding et al., 1995b; Ding et 


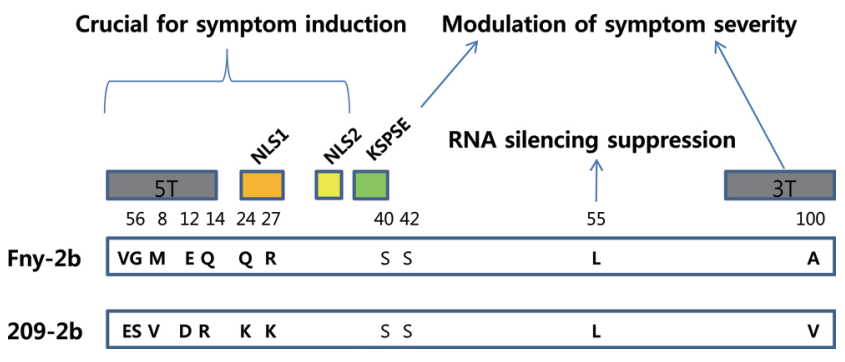

Fig. 5. Schematic map describing locations and roles of amino acids in the $2 \mathrm{~b}$ protein of CMV-Fny and -209. 5T, the first 17 amino acids of $2 \mathrm{~b}$ protein; NLS, arginine-rich nuclear localization sequence; KSPSE, the putative phosphorylation sequence; 3T, the $16 \mathrm{C}$-terminal residues of the $2 \mathrm{~b}$ protein. Numbers indicate amino acid residue position.

al., 1996; Du et al., 2007; Shi et al., 2002; Soards et al., 2002). Recently, the importance of specific domains within the $2 \mathrm{~b}$ protein for symptom induction has been investigated carefully (Ding et al., 1996; Goto et al., 2007; Lewsey et al., 2009). Lewsey et al. (2009) demonstrated that two nuclear localization signals (NLS1 and NLS2) and the $\mathrm{N}$-terminal domain (5T) of CMV-Fny $2 \mathrm{~b}$ protein were required and essential for symptom induction, respectively. The C-terminal domain (3T) and two serine residues within a putative phosphorylation domain (KSPSE) of CMV-Fny $2 \mathrm{~b}$ modulated symptom severity (Fig. 5). The comparison of amino acids sequence of $2 \mathrm{~b}$ protein of CMV-Fny and CMV-209 revealed that most of various amino acids were located at the N-terminal domain, two of them at NLS1 domain and one at C-terminal domain (Fig. 5). It is worth to determine which amino acid residues are responsible for symptom induction. Interestingly, although CMV209 caused latent symptom on $N$. benthamiana (Fig. 2B, 4B), the amino acid residues of the $2 \mathrm{~b}$ protein at position 55 being Leu was conserved in CMV-209 (Fig. 5), which has been reported to be crucial for the RNA silencing suppression and the induction of viral symptoms (Xu et al., 2013). It remains to be examined whether this conserved amino acid residue is actually responsible for suppressing silencing.

Reassortment of genomic segments is one of the mechanisms for genetic variation and new strain generation of multipartite RNA viruses (Morse, 1992). There is evidence that reassortment occurs in natural populations of plant virus (Chen et al., 2007; Fraile et al., 1997; Lin et al., 2004; Maoka et al., 2010). In spite of some studies demonstrated that the reassortment between CMV subgroups was a rare event (Bonnet et al., 2005; Fraile et al., 1997; Jacquemond, 2012), it did not mean that reassortment was not important in CMV evolution (Lin et al., 2004; Roossinck, 2002; White et al., 1995). For example, CMV-Tsh was a natural reassortant between CMV subgroup IA and II strains, in which RNAs 1 and 3 of CMV-Tsh was derived from one or two subgroup II strain(s), while RNA2 was derived from a subgroup I strain, revealed an evolution than its parents. It was probably that RNA2 of subgroup II was replaced by RNA2 of subgroup IA due to its low efficiency in suppression of host defense mechanism during the mixed infection of CMV-Tsh parental viruses (Chen et al., 2007). In the present work, we found that the reassortment between CMV-209 and CMV-Fny caused more severe symptom than wild-type strains (e.g. W1F2W3 and W1F2F3 inducing severe leaf distortion and stunting (Fig. 4E, G). In contrast, W1W2W3 showed latent symptom (Fig. 4B) and F1F2F3 exhibited mosaic, leaf distortion, and general stunting (Fig. 4C)). In addition, we also observed that a pseudorecombinant comprising of RNA1 of CMV-209 and RNAs 2 and 3 of CMV-12, an isolate form Azuki bean, showed more severe symptom than its parents (data not shown). These results indicated that natural reassortment between compatible viruses can result in emergence of novel viruses causing severe damages in crop fields.

\section{Acknowledgments}

This research was supported in part by grants from the Vegetable Breeding Research Center (No. 710001-03) through the Agriculture Research Center program from the Ministry for Food, Agriculture, Forestry and Fisheries; the Next-Generation BioGreen 21 Program (No. PJ00819801), Rural Development Administration (RDA); the Agenda Program (PJ00922904) funded by the RDA, Republic of Korea. MSVP was supported by a research fellowship from the Brain Korea 21 Plus Project.

\section{References}

Bonnet, J., Fraile, A., Sacristán, S., Malpica, J. M. and GarcíaArenal, F. 2005. Role of recombination in the evolution of natural populations of Cucumber mosaic virus, a tripartite RNA plant virus. Virology 332:359-368.

Brigneti, G., Voinnet, O., Li, W. X., Ji, L. H., Ding, S. W. and Baulcombe, D. C. 1998. Viral pathogenicity determinants are suppressors of transgene silencing in Nicotiana benthamiana. EMBO J. 17:6739-6746.

Chen, Y., Chen, J., Zhang, H., Tang, X. and Du, Z. 2007. Molecular evidence and sequence analysis of a natural reassortant between Cucumber mosaic virus subgroup IA and II strains. Virus Genes 35:405-413.

Davies, C. and Symons, R. H. 1988. Further implications for the 
evolutionary relationships between tripartite plant viruses based on Cucumber mosaic virus RNA 3. Virology 165:216224.

Ding, B., Li, Q., Nguyen, L., Palukaitis, P. and Lucas, W. J. 1995a. Cucumber mosaic virus 3a protein potentiates cellto-cell trafficking of CMV RNA in tobacco plants. Virology 207:345-353.

Ding, S.-W., Anderson, B. J., Haase, H. R. and Symons, R. H. 1994. New overlapping gene encoded by the Cucumber mosaic virus genome. Virology 198:593-601.

Ding, S.-W., Li, W.-X. and Symons, R. 1995b. A novel naturally occurring hybrid gene encoded by a plant RNA virus facilitates long distance virus movement. EMBO J. 14:5762-5772.

Ding, S.-W., Shi, B.-J., Li, W.-X. and Symons, R. H. 1996. An interspecies hybrid RNA virus is significantly more virulent than either parental virus. Proc. Natl. Acad. Sci. USA 93:7470-7474.

Divéki, Z., Salánki, K. and Balázs, E. 2004. The necrotic pathotype of the Cucumber mosaic virus (CMV) Ns strain is solely determined by amino acid 461 of the 1a protein. Mol. PlantMicrobe Interact. 17:837-845.

Du, Z.-Y., Chen, F.-F., Liao, Q.-S., Zhang, H.-R., Chen, Y.-F. and Chen, J.-S. 2007. 2b ORFs encoded by subgroup IB strains of Cucumber mosaic virus induce differential virulence on Nicotiana species. J. Gen. Virol. 88:2596-2604.

Du, Z., Chen, F., Zhao, Z., Liao, Q., Palukaitis, P. and Chen, J. 2008. The $2 \mathrm{~b}$ protein and the $\mathrm{C}$-terminus of the $2 \mathrm{a}$ protein of Cucumber mosaic virus subgroup I strains both play a role in viral RNA accumulation and induction of symptoms. Virology 380:363-370.

Fraile, A., Alonso-Prados, J. L., Aranda, M. A., Bernal, J. J., Malpica, J. M. and Garcia-Arenal, F. 1997. Genetic exchange by recombination or reassortment is infrequent in natural populations of a tripartite RNA plant virus. J. Virol. 71:934-940.

Fulton, J. P. 1950. Studies on strains of cucumber virus 1 from spinach. Phytopathology 40:729-736.

Gorbalenya, A. E., Koonin, E. V., Donchenko, A. P. and Blinov, V. M. 1989. Two related superfamilies of putative helicases involved in replication, recombination, repair and expression of DNA and RNA genomes. Nucleic Acids Res. 17:4713-4730.

Goto, K., Kobori, T., Kosaka, Y., Natsuaki, T. and Masuta, C. 2007. Characterization of silencing suppressor $2 \mathrm{~b}$ of Cucumber mosaic virus based on examination of its small RNAbinding abilities. Plant Cell Physiol. 48:1050-1060.

Habili, N. and Symons, R. H. 1989. Evolutionary relationship between luteoviruses and other RNA plant viruses based on sequence motifs in their putative RNA polymerases and nucleic acid helicases. Nucleic Acids Res. 17:9543-9555.

Hayes, R. J. and Buck, K. W. 1990. Complete replication of a eukaryotic virus RNA in vitro by a purified RNA-dependent RNA polymerase. Cell 63:363-368.

Hodgman, T. 1988. A new superfamily of replicative proteins. Nature 333:22-23.

Jacquemond, M. 2012. Cucumber mosaic virus. Adv. Virus Res
84:439-504

Kaplan, I. B., Shintaku, M. H., Li, Q., Zhang, L., Marsh, L. E. and Palukaitis, P. 1995. Complementation of virus movement in transgenic tobacco expressing the Cucumber mosaic virus 3a gene. Virology 209:188-199.

Lewsey, M., Robertson, F. C., Canto, T., Palukaitis, P. and Carr, J. P. 2007. Selective targeting of miRNA-regulated plant development by a viral counter-silencing protein. Plant J. 50:240-252.

Lewsey, M., Surette, M., Robertson, F. C., Ziebell, H., Choi, S. H., Ryu, K. H., Canto, T., Palukaitis, P., Payne, T. and Walsh, J. A. 2009. The role of the Cucumber mosaic virus $2 \mathrm{~b}$ protein in viral movement and symptom induction. Mol. Plant-Microbe Interact. 22:642-654.

Lin, H.-X., Rubio, L., Smythe, A. B. and Falk, B. W. 2004. Molecular population genetics of Cucumber mosaic virus in California: evidence for founder effects and reassortment. $J$. Virol. 78:6666-6675.

Lucy, A. P., Guo, H. S., Li, W. X. and Ding, S. W. 2000. Suppression of post-transcriptional gene silencing by a plant viral protein localized in the nucleus. EMBO J. 19:1672-1680.

Maoka, T., Hayano, Y. S., Iwasaki, M., Yoshida, K. and Masuta, C. 2010. Mixed infection in tomato to ensure frequent generation of a natural reassortant between two subgroups of Cucumber mosaic virus. Virus Genes 40:148-150.

Mi, S., Durbin, R., Huang, H. V., Rice, C. M. and Stollar, V. 1989. Association of the Sindbis virus RNA methyltransferase activity with the nonstructural protein nsP1. Virology 170:385-391.

Mi, S. and Stollar, V. 1991. Expression of sindbis virus nsP1 and methyltransferase activity in Escherichia coli. Virology 184:423-427.

Morse, S. S. 1992. Evolving views of viral evolution: towards an evolutionary biology of viruses. Hist. Philos. Life Sci. 14:215-248.

Palukaitis, P. and García-Arenal, F. 2003. Cucumoviruses. Adv. Virus Res. 62:241-323.

Palukaitis, P., Roossinck, M. J., Dietzgen, R. G. and Francki, R. 1992. Cucumber mosaic virus. Adv. Virus Res. 41:281-348.

Rao, A. and Francki, R. 1982. Distribution of determinants for symptom production and host range on the three RNA components of Cucumber mosaic virus. J. Gen. Virol. 61:197205.

Roossinck, M. J. 2002. Evolutionary history of Cucumber mosaic virus deduced by phylogenetic analyses. J. Virol. 76:33823387.

Rozanov, M. N., Koonin, E. V. and Gorbalenya, A. E. 1992. Conservation of the putative methyltransferase domain: a hallmark of the 'Sindbis-like'supergroup of positive-strand RNA viruses. J. Gen. Virol. 73:2129-2134.

Schwinghamer, M. W. and Symons, R. H. 1975. Fractionation of cucumber mosaic virus RNA and its translation in a wheat embryo cell-free system. Virology 63:252-262.

Seo, J.-K., Kwon, S.-J., Choi, H.-S. and Kim, K.-H. 2009. Evi- 
dence for alternate states of Cucumber mosaic virus replicase assembly in positive-and negative-strand RNA synthesis. Virology 383:248-260.

Shi, B.-J., Palukaitis, P. and Symons, R. H. 2002. Differential virulence by strains of Cucumber mosaic virus is mediated by the 2b gene. Mol. Plant-Microbe Interact. 15:947-955.

Shintaku, M. 1991. Coat protein gene sequences of two Cucumber mosaic virus strains reveal a single amino acid change correlating with chlorosis induction. J. Gen. Virol. 72:25872589.

Shintaku, M. H., Zhang, L. and Palukaitis, P. 1992. A single amino acid substitution in the coat protein of Cucumber mosaic virus induces chlorosis in tobacco. Plant Cell 4:751-757.

Soards, A. J., Murphy, A. M., Palukaitis, P. and Carr, J. P. 2002. Virulence and differential local and systemic spread of $\mathrm{Cu}$ cumber mosaic virus in tobacco are affected by the CMV $2 b$ protein. Mol. Plant-Microbe Interact. 15:647-653.

Suzuki, M., Kuwata, S., Masuta, C. and Takanami, Y. 1995. Point mutations in the coat protein of Cucumber mosaic virus affect symptom expression and virion accumulation in tobacco. $J$. Gen. Virol. 76:1791-1799.

Szilassy, D., Salánki, K. and Balázs, E. 1999. Stunting induced by cucumber mosaic cucumovirus-infected Nicotiana glutinosa is determined by a single amino acid residue in the coat protein. Mol. Plant-Microbe Interact. 12:1105-1113.
Takanami, Y. 1981. A striking change in symptoms on Cucumber mosaic virus-infected tobacco plants induced by a satellite RNA. Virology 109:120-126.

Troutman, J. L. and Fulton, R. W. 1958. Resistance in tobacco to Cucumber mosaic virus. Virology 6:303-316.

Vo Phan, M. S., Seo, J.-K., Choi, H.-S., Lee, S.-H. and Kim, K.-H. 2014. Molecular and biological characterization of an isolate of Cucumber mosaic virus from Glycine soja by generating its infectious full-genome cDNA clones. Plant Pathol. J. http://dx.doi.org/10.5423/PPJ.OA.02.2014.0014.

White, P. S., Morales, F. and Roossinck, M. J. 1995. Interspecific reassortment of genomic segments in the evolution of cucumoviruses. Virology 207:334-337.

Xu, A., Zhao, Z., Chen, W., Zhang, H., Liao, Q., Chen, J., Carr, J. P. and Du, Z. 2013. Self-interaction of the Cucumber mosaic virus $2 \mathrm{~b}$ protein plays a vital role in the suppression of RNA silencing and the induction of viral symptoms. Mol. Plant Pathol. 14:803-812.

Zhang, L., Handa, K. and Palukaitis, P. 1994. Mapping local and systemic symptom determinants of Cucumber mosaic cucumovirus in tobacco. J. Gen. Virol. 75:3185-3191.

Ziebell, H., Payne, T., Berry, J. O., Walsh, J. A. and Carr, J. P. 2007. A cucumber mosaic virus mutant lacking the $2 \mathrm{~b}$ counter-defence protein gene provides protection against wild-type strains. J. Gen. Virol. 88:2862-2871. 